Article

\title{
Cordon- or Link-Based Pricing: Environment-Oriented Toll Design Models Development and Application
}

\author{
Xijie Li ${ }^{1}$, Ying Lv ${ }^{1}$, , Wei Sun ${ }^{2}$ and Li Zhou ${ }^{3}$ \\ 1 Institute of Transportation System Science and Engineering, School of Traffic and Transportation, \\ Beijing Jiaotong University, Beijing 100044, China; 18120844@bjtu.edu.cn \\ 2 School of Geography and Planning, Sun Yat-Sen University, Guangzhou 510275, China; \\ sunwei29@mail.sysu.edu.cn \\ 3 School of Information, Beijing Wuzi University, Beijing 101149, China; zhoulibit@126.com \\ * Correspondence: ylv@bjtu.edu.cn
}

Received: 31 October 2018; Accepted: 25 December 2018; Published: 7 January 2019

check for updates

\begin{abstract}
This study focuses on an environment-friendly toll design problem, where an acceptable road network performance is promised. First, a Traffic Performance Index (TPI)-based evaluation method is developed to help identify the optimal congestion level and the management target of a transportation system. Second, environment-oriented cordon- and link-based road toll design models are respectively proposed through the use of bi-level programming. Both upper-level submodel objectives are to minimize gross revenue (the total collected toll minus the emissions treatment cost) under different pricing strategies. Both lower-level submodels quantify the user equilibrium (UE) condition under elastic demand. Moreover, the TPI-related constraints for the management requirements of the network performance are incorporated into the bi-level programming modeling framework, which can lead to $0-1$ mixed integer bi-level nonlinear programming for toll design problems. Accordingly, a genetic algorithm-based heuristic searching method is proposed for the two pricing models. The proposed cordon- and link-based pricing models were then applied to a real-world road network in Beijing, China. The effects of the toll schemes generated from the two models were compared in terms of emissions reduction and congestion mitigation. In this study, it was indicated that a higher total collected toll may lead to more emissions and related treatment costs. Tradeoffs existed between the toll scheme, emissions reduction, and congestion mitigation.
\end{abstract}

Keywords: cordon-based pricing; link-based pricing; toll design; congestion level; emission; bi-level programming

\section{Introduction}

The continued growth of motor vehicles associated with a large amount of exhaust emissions can lead to deteriorated ambient air quality, which is not ignored by urban authorities. Road transport, in particular, contributes significantly to most of the main air pollutants [1]. For example, in Beijing, China, motor vehicles were over 5.61 million, and the associated annual pollutant discharge was more than 700,000 tonnes, in 2016. Specifically, it was estimated that the pollutants contributed by motor vehicles accounted for $86 \%, 56 \%, 32 \%$, and $35.5 \%$ of carbon monoxide (CO), nitrogen oxides (NOx), hydrocarbons (HCs), and $\mathrm{PM}_{2.5}$ total emissions, respectively. Therefore, it is necessary to deal with the pollution problems caused by the urban traffic system.

Road pricing has been considered to be an effective measure for traffic demand management. It is essentially based on the principles of economics. Using economic leverage can internalize the externalities of the traffic system so that traffic flows can be reasonably distributed and the road resources can be optimized spatiotemporally. The road pricing theory was first proposed by Pigou 
(1920) and Knight (1924) in the 1920s, and since then many scholars have done a great deal of research work on the methods and technologies of road pricing [2-8]. For real-world pricing implementations, Singapore took the lead in implementing the first congestion pricing policy in the world in 1975, followed by other cities and areas such as London, Hong Kong, Oslo, Gothenburg, and Stockholm [9].

Generally, toll schemes can be classified in various ways, such as link-based [10], cordon-based [11], area-based [12], distance-based [13], and time-based schemes [14]. For example, Yang and Lam [15] proposed a bi-level programming approach for the determination of a coordinated link toll pattern, in which traffic assignment and road pricing were combined in general road networks. The link-based pricing aimed to seek the optimal tolls for the network links within a set of system constraints, and thus, such a toll scheme could be obtained with a certain degree of flexibility while satisfying various kinds of management requirements. However, link-based pricing is mostly a theoretical concept that is hard to implement due to the complexity of realistic road networks [16]. Cordon-based pricing allows vehicles to pay tolls to cross a cordon in the inbound direction, in the outbound direction, or possibly in both directions. Thus, the total number of vehicles that may enter the encircled area can be restricted. Cordon-based pricing is not necessary to determine toll locations and levels within the whole road network compared to the link-based one, which seems easier to implement in real-world management practices. Cordon-based pricing has been discussed, mainly focusing on optimal toll design [17], public acceptability [18], and policy analysis [19-21]. For example, Liu et al. [17] tackled the optimal toll design problem using a cordon-based congestion pricing scheme, where both a time toll and a nonlinear distance toll were levied for each network user's trip in a pricing cordon. Nevertheless, when environmental management requirements need to be addressed in the toll design problem, less attention has been paid to the impact comparison for further understanding the effectiveness of cordon- and link-based pricing strategies.

Since a tradeoff exists between system emissions and total travel time [22], more and more attention has gradually been paid to traffic emission-related pricing studies [23-27]. For example, Li et al. [23] proposed a two-stage road toll design model for congested road networks with physical and environmental capacity constraints, which could be used to create a sustainable urban transportation system. Ma et al. [26] proved that under certain conditions, there exists a free-flow optimal solution to minimize the generalized system cost, including total travel times and total emissions. Most previous studies have addressed environmental management targets through the formulation of minimal emission objectives or capacity constraints, and have used total travel time (TTT) to measure the congestion of a traffic system. However, TTT is not intuitive enough for real-world network management, because it may be difficult for local managers and travelers to understand actual congestion status while minimizing TTT.

Besides TTT, various indicators are also used for road network performance evaluations. They are developed based on non-uniform measurement methods by different agencies and are widely used around the world, such as the Roadway Congestion Index by the Texas Transportation Institute, calculated based on traffic density, the INRIX Index based on traffic speed, and several comprehensive indexes used in some Chinese cities (e.g., Beijing, Guangzhou, and Shenzhen) [28-31]. For example, the Traffic Performance Index (TPI) has been proposed as a comprehensive indicator by the Beijing Traffic Management Bureau (BTMB). It has obvious advantages in traffic state evaluation from a network perspective, since it takes road links associated with grades into account [32,33]. However, these indicators are currently used for traffic performance descriptions, and not enough attention has been paid to their interconnection with policy making for congestion improvement. Therefore, it is challenging to integrate a useful indicator into the optimization framework of the pricing model. The associated toll scheme needs to affect road network performance directly: Meanwhile, the feedback of the system evaluated by the indicator can result in the adjustment of management strategy.

The goal of this study was to develop environment-oriented road pricing models. Accordingly, TPI-based evaluation and optimization methods are proposed to help identify optimal performance and congestion levels. Then, cordon- and link-based toll design models are respectively developed 
through the use of bi-level programming under elastic demand. Meanwhile, TPI-related constraints are integrated to reflect congestion management requirements of the transportation system. Finally, the two pricing strategies are discussed based on applications to the road network of Beijing, China. The rest of the study is organized as follows: Section 2 presents evaluation and modeling of traffic performance and congestion levels. Section 3 formulates environment-oriented cordon- and link-based toll design models under elastic demand. Section 4 illustrates a numerical example. Section 5 describes the study case of Beijing, China. Section 6 shows results and discussions, and finally Section 7 concludes this study. In detail, Figure 1 shows the research framework of the study.

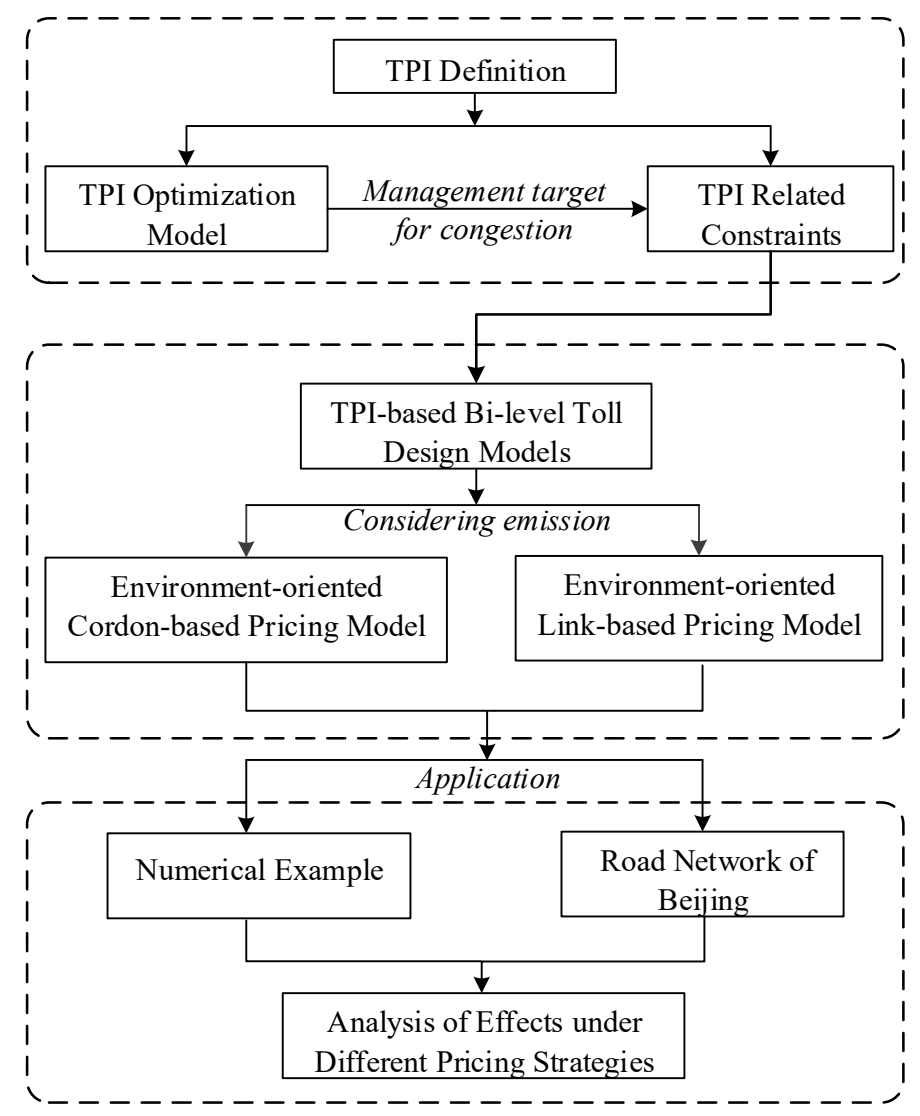

Figure 1. Research framework of the study.

\section{Traffic Performance and Congestion Level Evaluation and Modeling}

In order to assess road network performance, a TPI-based evaluation method is first introduced, a method for identifying optimal traffic performance and congestion level is developed, and TPI-related constraints for reflecting performance management requirements in pricing models are proposed in this section.

\subsection{Traffic Performance and Congestion Level Evaluation}

According to Reference [32], the TPI-based evaluation method can be described in the following steps.

\subsubsection{Identification of the Congested Road Link}

If the road link is congested, the average speed is less than the threshold of the link with a certain road grade, and thus we have

$$
v_{a, j}=\frac{l_{a, j}}{t_{a, j}}<\vartheta_{j}
$$


where $v_{a, j}$ is the average speed on link $a$ of road grade $j, l_{a, j}$ is the length of link $a$ of road grade $j$, $j=1,2,3,4$ (representing an expressway, arterial road, sub-arterial road, and branch road, respectively), $t_{a, j}$ is the travel time on link $a$ of road grade $j$, and $\vartheta_{j}$ is the speed threshold of road grade $j$. For an expressway, arterial road, sub-arterial road, and branch road, the threshold values are 20,15, 10, and $10 \mathrm{~km} / \mathrm{h}$, respectively.

\subsubsection{Calculation of the Network Congested Distance Proportion (CDP)}

In order to determine the TPI associated with road network performance and congestion level, the CDP value should be calculated first. The CDP is a weighted summation of the congested road links from each road grade in proportion to vehicle kilometers traveled (VKT). Thus, the CDP value can be obtained based on the following equation:

$$
C D P=\sum_{j=1}^{4} \frac{\sum_{a \in A_{j}} l_{a, j} k_{a, j} \sum_{a=A_{j}} x_{a, j} l_{a, j}}{\sum_{a \in A} l_{a, j} \cdot \sum_{j=1}^{4} \sum_{a \in A_{j}} x_{a, j} l_{a, j}},
$$

where $k_{a, j}$ equals 1 if $v_{a, j} \leq \vartheta_{j}$, and 0 otherwise; $A_{j}$ is the subset of $A$, representing the set of road grade $j$; and $x_{a, j}$ is traffic flow on link $a$ of road grade $j$.

\subsubsection{Determination of the TPI for the Performance and Congestion Level Evaluation}

Since the CDP has a corresponding conversion relationship with TPI, the TPI associated with the performance and congestion level of the road network can be obtained according to Table 1.

Table 1. The conversion relationship between congested distance proportion (CDP) and the Traffic Performance Index (TPI).

\begin{tabular}{ccc}
\hline CDP Value/\% & TPI & Performance and Congestion Level \\
\hline$[0,4]$ & {$[0,2]$} & A (Very smooth) \\
$(5,8]$ & $(2,4]$ & B (Smooth) \\
$(8,11]$ & $(4,6]$ & C (Lightly smooth) \\
$(11,14]$ & $(6,8]$ & D (Moderately congested) \\
$(14,24)$ & $(8,10)$ & E (Severely congested) \\
$\geq 24$ & 10 & E (Severely congested) \\
\hline
\end{tabular}

\subsection{Performance and Congestion Level Optimization Model}

Assume that the travel time on link $a$ can be presented by the Bureau of Public Roads (BPR) function of $t_{a}\left(x_{a}\right)=t_{a}^{0}\left(1+0.15\left(x_{a} / s_{a}\right)^{4}\right)$, where $t_{a}^{0}$ and $s_{a}$ represent the free-flow travel time (min) and the link capacity (vehicle/h), respectively. Based on Equation (1), we have

$$
k_{a, j}=\left\{\begin{array}{l}
1, v_{a, j}<\vartheta_{j} \\
0, v_{a, j} \geq \vartheta_{j}
\end{array}, a \in A, \forall j .\right.
$$

According to the relationship $v_{a, j}=l_{a, j} / t_{a, j}$, we also have

$$
k_{a, j}=\left\{\begin{array}{l}
1, x_{a, j}>\mu_{a, j} \\
0, x_{a, j} \leq \mu_{a, j}
\end{array},\right.
$$

where $\mu_{a, j}$ is the flow threshold of congested links, which can be obtained based on the BPR function and presented as $\mu_{a, j}=s_{a} \cdot\left(\left(\frac{l_{a, j}}{\vartheta_{a, j} \cdot t_{a}^{0}}-1\right) / 0.15\right)^{\frac{1}{4}}$. 
Let $\theta$ donate an arbitrary value between 0 and 1, and then Equation (4) can be expressed equivalently by the following constraint:

$$
\left(k_{a, j}-\theta\right)\left(x_{a, j}-\mu_{a, j}\right) \geq 0, k_{a, j} \in\{0,1\}, a \in A .
$$

Thus, the optimal performance and congestion level of a road network can be obtained through minimizing the CDP value, which can be provided as follows:

$$
\min \sum_{j=1}^{4} \frac{\sum_{a \in A_{j}} l_{a, j} k_{a, j} \sum_{a=A_{j}} x_{a, j} l_{a, j}}{\sum_{a \in A_{j}} l_{a, j} \cdot \sum_{j=1}^{4} \sum_{a \in A_{j}} x_{a, j} l_{a, j}}
$$

subject to

$$
\begin{gathered}
\left(k_{a, j}-\theta\right)\left(x_{a, j}-\mu_{a, j}\right) \geq 0, k_{a, j} \in\{0,1\}, a \in A, \\
\sum_{r} f_{r}^{w}=d_{w}, w \in W, \\
x_{a, j}=\sum_{w \in W} \sum_{r \in R_{w}} f_{r}^{w} \delta_{a r}^{w}, a \in A, \\
f_{r}^{w} \geq 0, r \in R_{w}, w \in W .
\end{gathered}
$$

Apparently, Model (6) can generate the optimal performance and congestion level and help identify a reasonable management requirement for road network performance.

\subsection{Formulation of TPI-Related Constraints}

Based on the outputs of Model (6), the management requirement for road network performance can be identified accordingly. It can be described as the following TPI-related constraints:

$$
\begin{gathered}
\sum_{j=1}^{4} \frac{\sum_{a \in A_{j}} l_{a, j} k_{a, j} \sum_{a=A_{j}} x_{a, j} l_{a, j}}{\sum_{a \in A_{j}} l_{a, j} \cdot \sum_{j=1}^{4} \sum_{a \in A_{j}} x_{a, j} l_{a, j}} \leq \bar{\rho}_{m^{\prime}} \\
\left(k_{a, j}-\theta\right)\left(x_{a, j}-\mu_{a, j}\right) \geq 0, k_{a, j} \in\{0,1\}, a \in A,
\end{gathered}
$$

where $\bar{\rho}_{m}$ is the upper bound of the CDP when the network under the performance and congestion level is $m, m \in\{A, B, C, D, E\}$. For example, when the management target is set to level $m$ (with a CDP range of $\left[\underline{\rho}_{m}, \bar{\rho}_{m}\right]$ ), the parameter of $\bar{\rho}_{m}$ in the right-hand side of Equation (7a) thus can be identified according to Table 1 . The road network performance should be no worse than level $m$. Then Equations (7a) and ( $7 \mathrm{~b})$ can be integrated into the toll design models to reflect the requirement for the road network performance.

\section{Development of the Environment-Oriented Toll Design Models}

\subsection{Environment-Oriented Cordon-Based Toll Design Model}

For a traffic network, the user compares and chooses the shortest path based on the User Equilibrium (UE) principle, when the generalized link cost is changed by the local manager. Thus, the decision process between the manager and the user can be described using the following bi-level programming (BLP) model [34].

The upper-level submodel is

$$
\min _{\mathbf{x}} F(\mathbf{x}, \mathbf{y})
$$


subject to

$$
\mathbf{G}(\mathbf{x}, \mathbf{y}) \leq \mathbf{0}
$$

The lower-level submodel is

$$
\min _{\mathbf{y}} f(x, y)
$$

subject to

$$
\mathbf{g}(\mathbf{x}, \mathbf{y}) \leq \mathbf{0},
$$

where $\mathbf{x}$ and $\mathbf{y}$ are the decision vectors of the upper- and lower-level submodels, respectively; and $\mathbf{y}=\mathbf{y}(\mathbf{x})$ is the reaction or response function, which can be obtained by the lower-level submodel and used to replace $\mathbf{y}$ in the upper-level one.

Accordingly, the BLP method can be applied to the cordon-based toll design problem, wherein the local manager considers both toll collections and emission externalities in the upper-level submodel and the UE condition under elastic demand in the lower-level submodel. Thus, a cordon-based bi-level programming toll design model can be formulated as follows.

The upper-level submodel is

$$
\begin{gathered}
\min _{\tau} \sum_{a \in A_{c}} \tau_{a, j} x_{a, j}(\tau)-\mu \sum_{a \in A_{k}} e_{a, j}\left(x_{a, j}(\tau)\right) x_{a, j}(\tau), \\
\tau_{\min }^{c} \leq \tau_{a, j} \leq \tau_{\max }^{c}, \forall a \in A_{c}, \\
\sum_{a \in A_{c}} \tau_{a, j} x_{a, j}(\tau)-\mu \sum_{a \in A_{k}} e_{a, j}\left(x_{a, j}(\tau)\right) x_{a, j}(\tau) \geq 0,
\end{gathered}
$$

where $x_{a, j}(\tau)$ represents flow on link $a$ of grade $j$ and is the solution to the lower-level problem of UE under elastic demand.

The lower-level submodel is

$$
\begin{gathered}
\min Z\left(x_{a}, d_{w}\right)=\sum_{a \in A_{k}} \int_{0}^{x_{a}(\tau)} c_{a}\left(\omega, \tau_{a}\right) d \omega-\sum_{w \in W_{k}} \int_{0}^{d_{w}} D_{w}^{-1}(\omega) d \omega, \\
\sum_{r} f_{r}^{w}=d_{w}, w \in W, \\
x_{a, j}=\sum_{w \in W} \sum_{r \in R_{w}} f_{r}^{w} \delta_{a r}^{w}, a \in A, \\
f_{r}^{w} \geq 0, r \in R_{w}, w \in W,
\end{gathered}
$$

where $\tau_{a, j}$ is a toll on link $a ; A_{c} \subset A$ is the subset of links subjected to a cordon toll; $A_{k} \subset A$ is the subset of links subjected to a link toll; $\mu$ is the unit cost of emission; $\tau_{\max }^{c}$ and $\tau_{\min }^{c}$ are the upper and lower limits of $\tau_{a, j}$ for the cordon toll; $W$ is the set of origin-destination (O-D) pairs of the system; $W_{k}$ is the subset of O-D pairs within the tolled area, and $W_{k} \subset W ; d_{w}$ represents the demand between O-D pair $w, w \in W ; R_{w}$ is the set of paths connecting O-D pair $w ; \delta_{a r}^{w}$ equals 1 if route $r$ between O-D pair $w$ uses link $a, a \in A$, and 0 otherwise; $t_{a, j}\left(x_{a, j}\right)$ is travel time on link $a$; and $f_{r}^{w}$ represents traffic flow on path $r$. Furthermore, the generalized link travel cost (including both travel time and toll charge, if any) is given by $c_{a}\left(x_{a, j}, \tau_{a, j}\right)=t_{a}\left(x_{a, j}\right)+\tau_{a, j}$.

Through the introduction of the TPI-related constraints proposed in Section 2, an environment-oriented cordon-based bi-level toll design model can be formulated under elastic demand, as follows.

In the upper-level submodel, the objective is to minimize gross revenue. The function includes the total collected tolls and the emissions treatment cost, and can be provided as follows:

$$
\min _{\tau} \sum_{a \in A_{c}} \tau_{a, j} x_{a, j}(\tau)-\mu \sum_{a \in A_{k}} e_{a, j}\left(x_{a, j}(\tau)\right) x_{a, j}(\tau),
$$


where $e_{a, j}\left(x_{a, j}(\tau)\right)$ is the carbon monoxide (CO) emission on link $a$ of road grade $j(\mathrm{~g})$. In this study, we considered $\mathrm{CO}$ to be a representative indicator of vehicle exhaust emission, and $e_{a, j}\left(x_{a, j}(\tau)\right)$ can be approximatively presented by $e_{a, j}\left(x_{a, j}\right)=4.23605 \times 10^{-15} \times l_{a, j} \times x_{a, j}^{4}+0.45184 \times l_{a, j}[35]$, subject to the following:

(1) TPI-related constraints,

$$
\begin{gathered}
\sum_{j=1}^{4} \frac{\sum_{a \in A_{j}} l_{a, j} k_{a, j} \sum_{a=A_{j}} x_{a, j} l_{a, j}}{\sum_{a \in A_{j}} l_{a, j} \cdot \sum_{j=1}^{4} \sum_{a \in A_{j}} x_{a, j} l_{a, j}} \leq \bar{\rho}_{m}, A_{j} \subseteq A_{k} \\
\left(k_{a, j}-\theta\right)\left(x_{a, j}-\mu_{a, j}\right) \geq 0, k_{a, j} \in\{0,1\}, a \in A_{k} ;
\end{gathered}
$$

(2) Toll restrictions,

$$
\tau_{\min }^{c} \leq \tau_{a, j} \leq \tau_{\max }^{c}, \forall a \in A_{c},
$$

where $\tau_{a, j}$ is a toll on link $a$ of road grade $j$; and

(3) Gross revenue balance,

$$
\sum_{a \in A_{c}} \tau_{a, j} x_{a, j}(\tau)-\mu \sum_{a \in A_{k}} e_{a, j}\left(x_{a, j}(\tau)\right) x_{a, j}(\tau) \geq 0
$$

In the lower-level submodel, this submodel describes the UE problem under elastic demand, which can be presented as follows,

$$
\min Z\left(x_{a}, d_{w}\right)=\sum_{a \in A_{k}} \int_{0}^{x_{a}(\tau)} c_{a}\left(\omega, \tau_{a}\right) d \omega-\sum_{w \in W_{k}} \int_{0}^{d_{w}} D_{w}^{-1}(\omega) d \omega
$$

subject to the following:

(4) The relationship between path flow and O-D demand,

$$
\sum_{r} f_{r}^{w}=d_{w}, w \in W
$$

(5) The relationship between flow on link $a$ and path flow (use link $a$ ),

$$
x_{a, j}=\sum_{w \in W} \sum_{r \in R_{w}} f_{r}^{w} \delta_{a r}^{w}, a \in A
$$

(6) Non-negative constraint of path flow,

$$
f_{r}^{w} \geq 0, r \in R_{w}, w \in W .
$$

In the above model, the demand function can be expressed as $d_{w}=\bar{d}_{w} \cdot e^{\kappa \cdot \mu_{w}}, w \in W_{k}$, where $\bar{d}_{w}$ is the potential demand for the O-D pair, and $\kappa$ is the parameter reflecting the sensitivity of users to the generalized travel cost. Consequently, the toll scheme (decision variable of $\tau_{a, j}$ ) associated with the traffic flow $x_{a, j}$ can be obtained through solving the above BLP model.

\subsection{Environment-Oriented Link-Based Toll Design Model}

In comparison, the link-based toll design model has a different toll pattern from the cordon-based one. It considers links within the tolled area as candidate charging locations. Although the model expression is similar to the previous cordon-based model, the specific meanings of the model 
expressions may not be the same. Thus, the environment-oriented link-based toll design model can be presented as follows.

The objective of the upper-level submodel is also to minimize the gross revenue, wherein the tolls can be collected from the candidate links within the tolled area:

$$
\min _{\tau} \sum_{a \in A_{k}} \tau_{a, j} x_{a, j}(\tau)-\mu \sum_{a \in A_{k}} e_{a, j}\left(x_{a, j}(\tau)\right) x_{a, j}(\tau),
$$

subject to the following:

$$
\begin{gathered}
\sum_{j=1}^{4} \frac{\sum_{a \in A_{j}} l_{a, j} k_{a, j} \sum_{a=A_{j}} x_{a, j} l_{a, j}}{\sum_{a \in A_{j}} l_{a, j} \cdot \sum_{j=1}^{4} \sum_{a \in A_{j}} x_{a, j} l_{a, j}} \leq \bar{\rho}_{m^{\prime}}, A_{j} \subseteq A_{k}, \\
\left(k_{a, j}-\theta\right)\left(x_{a, j}-\mu_{a, j}\right) \geq 0, k_{a, j} \in\{0,1\}, a \in A_{k}, \\
\tau_{\min } \leq \tau_{a, j} \leq \tau_{\max }, \forall a \in A_{k \prime} \\
\sum_{a \in A_{k}} \tau_{a, j} x_{a, j}(\tau)-\mu \sum_{a \in A_{k}} e_{a, j}\left(x_{a, j}(\tau)\right) x_{a, j}(\tau) \geq 0,
\end{gathered}
$$

where $\tau_{\max }^{c}$ and $\tau_{\min }^{c}$ are the upper and lower limits of $\tau_{a, j}$ for the link toll.

The lower-level submodel describes the UE problem under elastic demand, which can be presented as follows:

$$
\min Z\left(x_{a, j}, d_{w}\right)=\sum_{a \in A_{k}} \int_{0}^{x_{a, j}(\tau)} c_{a, j}\left(\omega, \tau_{a, j}\right) d \omega-\sum_{w \in W_{k}} \int_{0}^{d_{w}} D_{w}^{-1}(\omega) d \omega .
$$

In Equation (11f), the elastic demands refer to all the O-D pairs within the tolled area, which are different from the cordon-based toll design model (existing in the access links to the cordon). Moreover, the constraints of the lower-level submodel have similar expressions to those of the cordon-based toll design model, as shown in Equations (10g)-(10i).

Both the cordon- and link-based toll design methods are based on 0-1 mixed integer bi-level nonlinear programming models. Accordingly, genetic algorithm-based heuristic solution methods are proposed for both pricing models.

\section{Numerical Example}

In this section, we present a hypothetical network for the numerical example to illustrate the applications of the proposed methods, which are shown in Figure 2. It consists of two O-D pairs, $(1,9)$ and $(2,10)$. The link travel time could be estimated by using the BPR function. Moreover, the tolled locations for the cordon-based model are Links 15 and 16 , with $A_{c}=\{15,16\}$. The set of the candidate tolled links for the link-based model is $A_{k}=\{1,2,3,4,5,6,7,8,9,10,11,12,13,14\}$. Additionally, the detailed parameters used in the numerical example are presented in Table 2. 


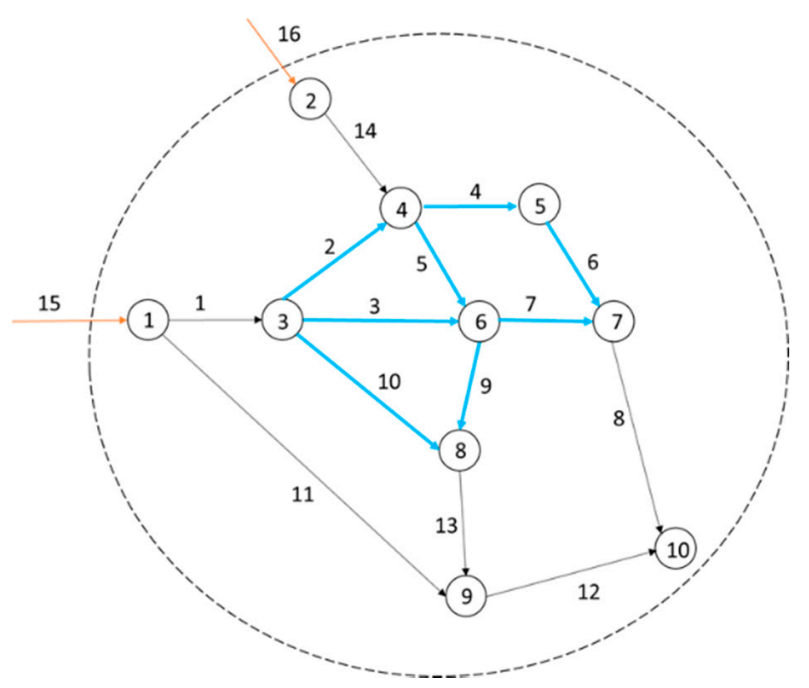

Figure 2. The road network of the numerical example.

Table 2. Related parameters of the road network.

\begin{tabular}{cccccc}
\hline Links & Road Grade $j$ & Speed Threshold $\vartheta_{j}$ & $\boldsymbol{l}_{\boldsymbol{a}} \mathbf{( \mathbf { k m } )}$ & $\boldsymbol{t}_{\boldsymbol{a}, \boldsymbol{j}}^{\mathbf{0}}(\mathbf{m i n})$ & $\boldsymbol{s}_{\boldsymbol{a}}(\mathbf{v e h i c l e} / \mathbf{h})$ \\
\hline 1 & 2 & 15.00 & 0.40 & 0.40 & 3000.00 \\
2 & 1 & 20.00 & 0.35 & 0.35 & 2000.00 \\
3 & 1 & 20.00 & 0.80 & 0.80 & 2000.00 \\
4 & 1 & 20.00 & 0.30 & 0.30 & 2000.00 \\
5 & 1 & 20.00 & 0.40 & 0.40 & 2500.00 \\
6 & 1 & 20.00 & 0.55 & 0.55 & 2000.00 \\
7 & 1 & 20.00 & 0.30 & 0.30 & 2000.00 \\
8 & 2 & 15.00 & 0.60 & 0.60 & 2000.00 \\
9 & 1 & 20.00 & 0.30 & 0.30 & 3000.00 \\
10 & 1 & 20.00 & 0.90 & 0.90 & 2000.00 \\
11 & 2 & 15.00 & 1.80 & 1.80 & 2500.00 \\
12 & 2 & 15.00 & 0.40 & 0.40 & 2000.00 \\
13 & 2 & 15.00 & 0.30 & 0.30 & 3500.00 \\
14 & 2 & 15.00 & 0.60 & 0.60 & 3000.00 \\
15 & $/$ & $/$ & 4.8 & 4.8 & 3000.00 \\
16 & $/$ & $/$ & 5.46 & 5.46 & 3000.00 \\
\hline
\end{tabular}

In order to compare the effects of the cordon- and link-based pricing methods, several rules were regulated as follows:

(1) The area within the cordon (the dotted line) shown in Figure 2 was considered to be a controlled area by toll schemes, and the regional effects in terms of emissions reduction and congestion mitigation were investigated and compared under pricing strategies;

(2) Both the cordon- and link-based toll design models had the same O-D pairs and expressions of elastic demand. Meanwhile, the other parameters of the road network were the same;

(3) In spite of different tolled locations, the total available collected tolls (the sum of the upper limits of tolls) were the same. For example, the cordon- and link-based tolls had 2 and 14 tolled locations (or links), and the upper limits of the collected tolls were 7 and 1 for the cordon- and link-based pricing, respectively. Thus, the total available collected tolls from the road network was 14 for the two pricing strategies.

Without policy intervention, the CDP could be calculated under the UE condition with a value of $9.73 \%$. Thus, the congestion level of the road network belonged to level C (lightly smooth). In order to make a reasonable management target for congestion control under pricing strategies, the TPI-based evaluation model (Model (6)) needed to first be solved. Therefore, the network 
performance management target could be predefined based on the optimal congestion level of "very smooth", and the right-hand side parameter of Inequality (7a) (the TPI-related constraints) could be determined with a value of $4 \%$ for the two pricing models (Models (10) and (11)).

The results obtained from the two models are presented in Tables 3 and 4. The total travel demands could be reduced from the original potential demand of 13,500 to $12,686.91$ and $12,688.47$ under the cordon- and link-based pricing, respectively. Without policy intervention (i.e., under the UE condition), the total travel time and regional emissions were $51,736.52 \mathrm{~min}$ and $64.09 \mathrm{~kg}$, respectively. Thus, they both decreased under the pricing strategies. Comparatively, the travel time in the control area was higher under the link-based pricing than under the cordon-based one. In spite of more regional emissions than under link-based pricing, the pollution treatment cost could be covered by the higher total toll collected by the cordon-based pricing.

Table 3. Origin-destination (O-D) demands under different policy scenarios.

\begin{tabular}{cccc}
\hline O-D Pairs & $\begin{array}{c}\text { The Expression of } \\
\text { Elastic Demand }\end{array}$ & Cordon-Based Pricing & Link-Based Pricing \\
\hline$(1,9)$ & $6500 \cdot e^{-0.01 \cdot u_{1,9}}$ & 6342.68 & 6347.34 \\
$(2,10)$ & $7000 \cdot e^{-0.01 \cdot u_{2,10}}$ & 6344.23 & 6341.13 \\
Total travel demand & & $12,686.91$ & $12,688.47$ \\
\hline
\end{tabular}

Table 4. Solutions to the cordon- and link-based pricing models.

\begin{tabular}{|c|c|c|c|c|c|c|c|}
\hline \multirow{2}{*}{ Links } & \multirow{2}{*}{ Road Grade $j$} & \multicolumn{3}{|c|}{ Cordon-Based Pricing } & \multicolumn{3}{|c|}{ Link-Based Pricing } \\
\hline & & Flow & Toll & Congestion Level & Flow & Toll & Congestion Level \\
\hline 1 & 2 & 3563.82 & / & A & 3355.13 & 0.00 & A \\
\hline 2 & 1 & 0.00 & / & $\mathrm{B}$ & 0.00 & 0.34 & B \\
\hline 3 & 1 & 1233.41 & / & B & 753.36 & 0.00 & $\mathrm{~B}$ \\
\hline 4 & 1 & 1956.78 & / & $\mathrm{B}$ & 2390.38 & 0.00 & $\mathrm{C}$ \\
\hline 5 & 1 & 4387.44 & / & $\mathrm{D}$ & 3950.75 & 0.00 & $\mathrm{D}$ \\
\hline 6 & 1 & 1956.78 & / & $\mathrm{B}$ & 2390.38 & 0.00 & $\mathrm{C}$ \\
\hline 7 & 1 & 1775.30 & / & $\mathrm{B}$ & 1818.03 & 0.00 & $\mathrm{~B}$ \\
\hline 8 & 2 & 3732.09 & / & $\mathrm{C}$ & 4208.41 & 0.01 & $\mathrm{D}$ \\
\hline 9 & 1 & 3845.55 & / & $\mathrm{C}$ & 2886.09 & 0.13 & $\mathrm{~B}$ \\
\hline 10 & 1 & 2330.41 & / & $\mathrm{C}$ & 2601.77 & 0.00 & $\mathrm{C}$ \\
\hline 11 & 2 & 2778.87 & / & A & 2992.20 & 0.00 & A \\
\hline 12 & 2 & 2612.14 & / & A & 2132.72 & 1.00 & A \\
\hline 13 & 2 & 6175.96 & / & $\mathrm{C}$ & 5487.85 & 0.00 & B \\
\hline 14 & 2 & 6344.23 & / & $\mathrm{D}$ & 6341.13 & 1.00 & $\mathrm{D}$ \\
\hline 15 & / & 6342.68 & 0.00 & / & 6347.34 & / & / \\
\hline 16 & / & 6344.23 & 4.37 & / & 6341.13 & / & / \\
\hline \multicolumn{2}{|c|}{ Total Travel time (min) } & \multicolumn{3}{|c|}{$47,617.44$} & \multicolumn{3}{|c|}{$49,248.00$} \\
\hline \multicolumn{2}{|c|}{ Total emissions $(\mathrm{g})$} & \multicolumn{3}{|c|}{$56,690.31$} & \multicolumn{3}{|c|}{$51,639.38$} \\
\hline
\end{tabular}

\section{Applications to Beijing, China}

In this section, both the cordon- and link-based toll design models were applied to the Beijing 2nd Ring Road network (Figure 3). The effects of the two pricing strategies on emission reduction and congestion mitigation were investigated and are discussed. 


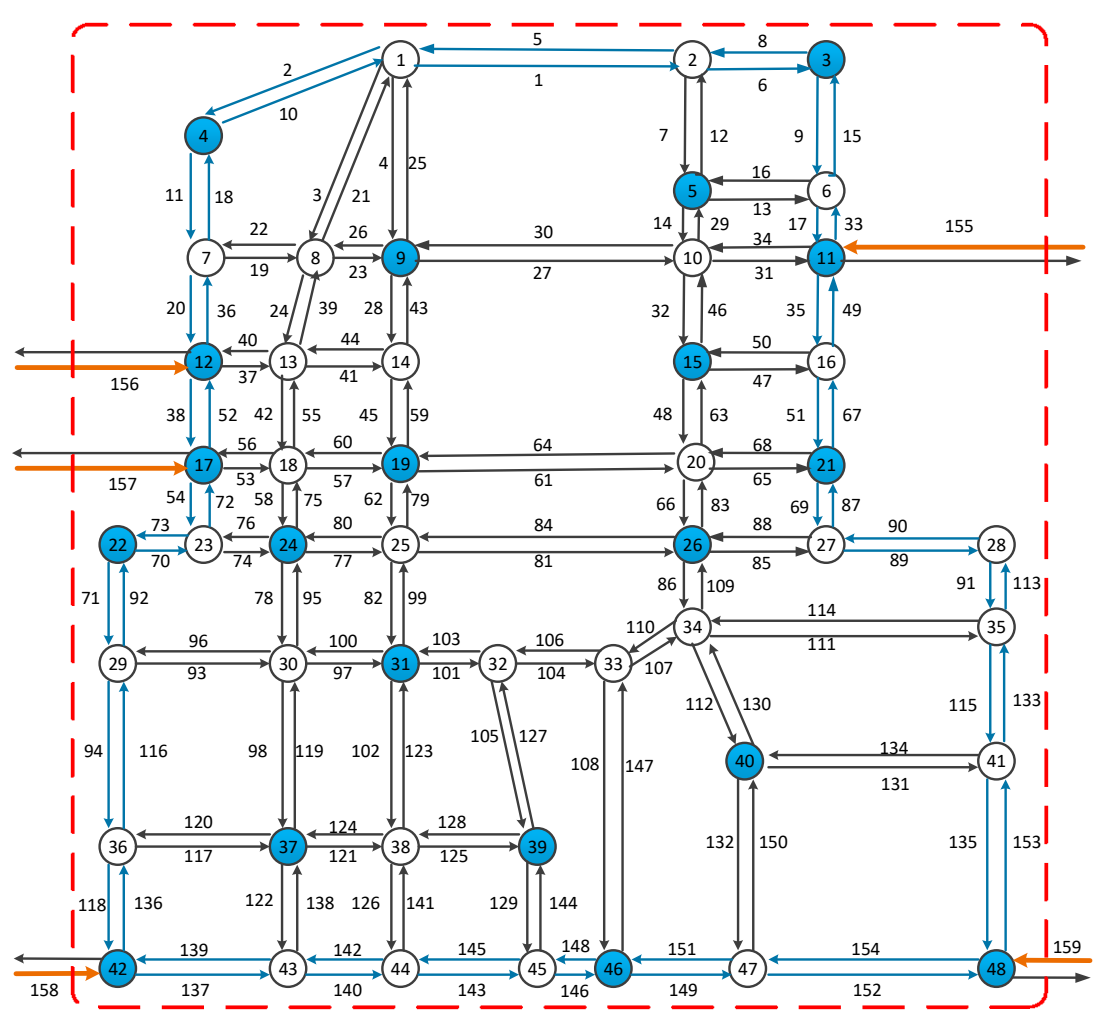

Figure 3. Beijing 2nd Ring Road network topology.

People living in megacities around the world, such as Beijing, are always eager to travel in efficient and environmentally friendly ways. However, people in Beijing suffer from air pollution and traffic congestion, which are partly caused by the urban transportation system. In 2017, the TPI of the Beijing 2nd Ring Road was 7.0, an increase of $2.6 \%$ over last year, during which the congestion level was "moderately congested" [36]. Therefore, it is urgent to take effective measures to improve environment and congestion issues. The 2nd Ring Road network is a typical study area of Beijing, with desired demands for emission control and congestion management. Moreover, the transportation infrastructures in the area can be well-developed, which is the foundation of implementing the pricing strategy. Figure 3 shows the topological graph of the study network. The area inside the red dotted line is the region where the pricing strategy could be applied. In detail, the cordon-based tolls could be charged at the access links (i.e., Links 155-159), and the link-based tolls could be charged inside the cordon with the candidate tolled locations of Links 1-154. The blue nodes in the figure represent the origin and destination points. Similarly, the rules proposed in Section 4 were also employed for comparison of the effects of both pricing strategies.

\section{Results and Discussion}

For the study road network, the traffic flow distribution associated with the network CDP value under the UE condition was also calculated using the system input parameters, which could represent traffic status without policy intervention. The results showed that under the UE condition, the CDP value was $6.44 \%$, which corresponded to the road network performance of level B (smooth).

Furthermore, the optimal performance and congestion level of the road network were identified through the CDP optimization model, and thus, the TPI-related constraints could be formulated to reflect the road network management target. It was indicated that the optimal CDP value obtained from Model (6) corresponded to the performance level of A based on Table 1. Accordingly, the right-hand side parameters of $\bar{\rho}_{A}$ in constraints (10b) and (11b), respectively, for the cordon- and link-based toll design models, were $4 \%$ for the study network. Then, the toll schemes could be generated from the 
two pricing models. In detail, the tolls charged at the links accessing the cordon for the cordon-based pricing strategy are provided in Table 5, and the toll scheme for the link-based one is presented in Figure 4. Correspondingly, the total practical travel demands for the UE, cordon-, and link-based models were $201,968.9,163,981$, and 199,804, respectively.

Table 5. Toll scheme from cordon-based pricing.

\begin{tabular}{cccccc}
\hline Link & $\mathbf{1 5 5}$ & $\mathbf{1 5 6}$ & $\mathbf{1 5 7}$ & $\mathbf{1 5 8}$ & $\mathbf{1 5 9}$ \\
\hline Toll (min) & 61.50 & 61.60 & $\begin{array}{c}61.60 \\
807,010.70\end{array}$ & 40.21 & 0.00 \\
Total collected toll (min) & & & & \\
\hline
\end{tabular}

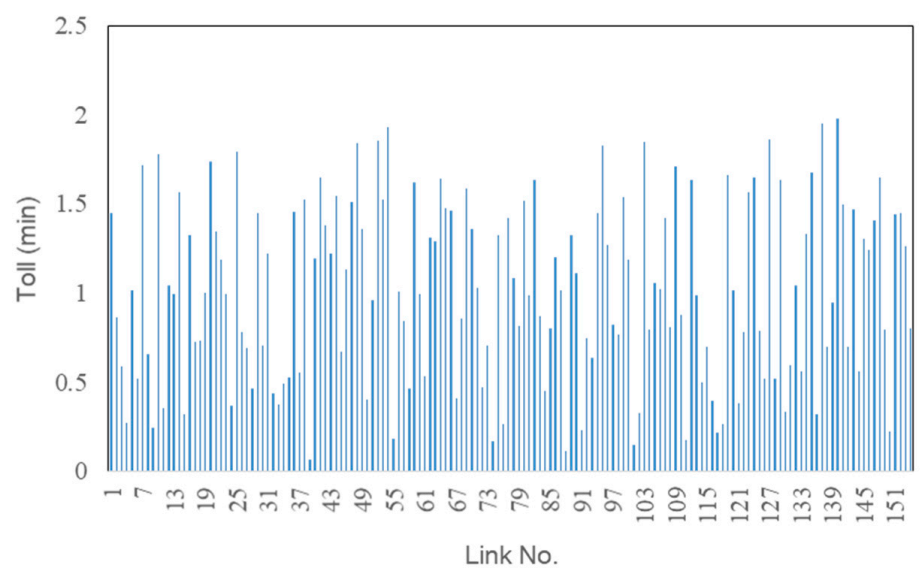

Figure 4. Toll scheme from link-based pricing.

Moreover, the results of the TPI evaluation, the TTT, the regional total emissions, and the total collected tolls of the study case, could be investigated and compared between the two pricing strategies, which are summarized in Figure 5.

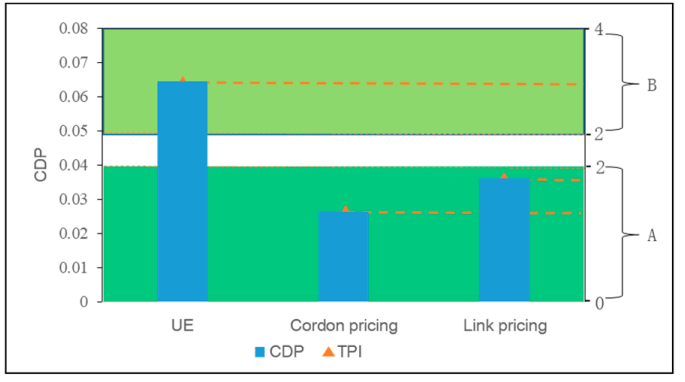

(a)

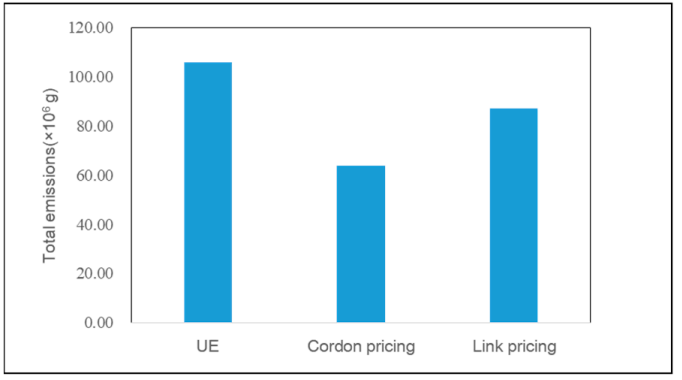

(c)

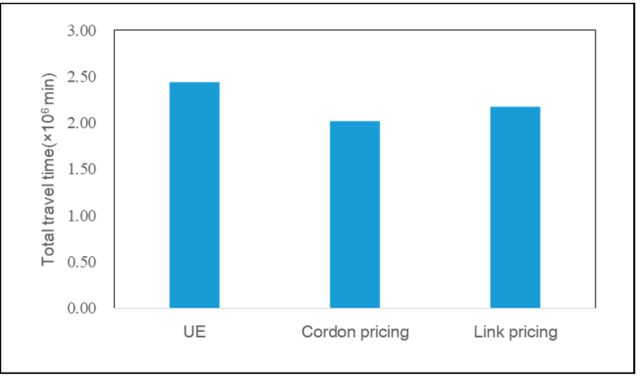

(b)

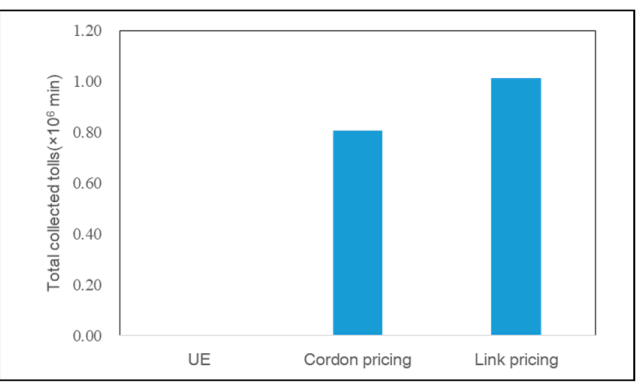

(d)

Figure 5. Result comparisons under different policy scenarios: (a) CDP value for TPI-based road performance evaluation; (b) total travel time (TTT); (c) total emissions; and (d) total collected tolls. 
From the perspective of road network performance management, congestion levels are evaluated based on CDP values under different policy scenarios. In detail, the CDP values obtained from Models (10) and (11) were lower than those without policy intervention. They decreased from $6.44 \%$ under the UE condition to $2.64 \%$ under cordon-based pricing and $3.61 \%$ under link-based pricing. Accordingly, the congestion level improved from level B to A. The network performance could achieve a very smooth status while pricing. Moreover, TTT also decreased under pricing strategies, and was reduced from $2.44 \times 10^{6} \mathrm{~min}$ under the UE condition to $2.02 \times 10^{6} \mathrm{~min}$ and $2.18 \times 10^{6} \mathrm{~min}$ with the cordonand link-based pricing.

Since total practical travel demand could be largely reduced by the cordon-based toll scheme, the related TTT was much lower than with the link-based one. In order to further understand the environmental effect of the pricing policies on the study system, the total emissions of the tolled area were also investigated under different management scenarios. In the study case, the total emissions were $105.92 \times 10^{3} \mathrm{~kg}$ under the UE condition. It was indicated that total emissions could be effectively reduced by the pricing policies as well. Through implementing the cordon-based pricing strategy, the related total emissions within the tolled area decreased to $63.85 \times 10^{3} \mathrm{~kg}$, with a reduction of $39.7 \%$. As for the link-based pricing strategy, the total emissions were reduced by $17.8 \%$, corresponding to a total of $87.08 \times 10^{3} \mathrm{~kg}$ in vehicle exhaust. The pricing policies focusing on emissions controls of the tolled area may lead to rescheduling routes and potential increases in traffic flows in the links outside the tolled area. For example, partial vehicles would be forced not to enter the tolled area under cordon-based pricing. The results showed that the total emissions of the study system did not increase under the cordon- and link-based polices. The emissions were respectively reduced to $63.94 \times 10^{3} \mathrm{~kg}$ and $87.32 \times 10^{3} \mathrm{~kg}$ through the cordon- and link-based schemes compared to the UE condition $\left(106.17 \times 10^{3} \mathrm{~kg}\right)$. Both pricing strategies played positive roles in pollution mitigation. Comparatively, the cordon-based pricing was better than the link-based one in terms of emissions reduction in the study network.

In the study, the revenue from the toll collection was mainly used for emissions treatment. Thus, the pricing strategy with a higher emissions level may increase the total collected toll. For example, the emissions amount under the link-based pricing was higher than under the cordon one, which would thus lead to more emissions treatment costs. Accordingly, in order to cover the emissions treatment costs through pricing, the total collected toll under the link-based pricing needed to be increased. In detail, the sum of the tolls charged at the links accessing the cordon was $807.01 \times 10^{3}$ under the cordon-based pricing strategy for the study area. As for the link-based pricing strategy, a relatively higher total collected toll of $1013.83 \times 10^{3}$ was obtained, comparatively.

Overall, the differences between the cordon- and link-based pricing strategies rely on both the charging mechanism and the effect in terms of emissions and congestion improvements. From the aspect of a charging mechanism, the cordon-based pricing only charges travelers who use the links to access the cordon, and the link-based one considers all the candidate links within the tolled area and determines the associated toll levels, which may be more complicated to implement in real-world management practice. Moreover, the cordon toll scheme influences a traveler's decision, and may reduce travel demand for entering the tolled area, while the link-based one requires reallocation of traffic flows under travel demand. Thus, for the numerical example, the cordon toll scheme could lead to a flow pattern with less TTT, and for the real-world network with less practical travel demand, the decrease of TTT was more significant compared to the link-based pricing. In terms of the improvement in emissions abatement, it depends on not only the travel demand, but also the toll design (the toll locations and levels), which can be investigated in a future study. The factors thus influence the total collected toll. In the study, the collected toll was mainly used to cover emissions treatment costs. Under a higher total collected toll level, although it leads to more emissions, the related treatment costs can be guaranteed. Thus, a tradeoff exists between the toll scheme, emissions reduction, and congestion mitigation. 


\section{Conclusions}

This paper studied environmentally friendly pricing strategies where an acceptable road network performance is promised. First, a TPI-based evaluation method was proposed in order to help identify the optimal performance and congestion level of the road network. Then, environment-oriented cordonand link-based toll design models were respectively proposed through incorporation of TPI-related constraints to reflect the performance management target of the traffic system. Both toll design models were developed based on bi-level programming frameworks. In detail, the upper-level submodel objectives are to minimize gross revenue, including the total collected tolls and the emissions treatment costs under different pricing strategies; and the lower-level submodels consider UE conditions under elastic demand.

A numerical example was used to demonstrate the applications of the developed methods. Meanwhile, the proposed cordon- and link-based pricing models were applied to a real-world road network in Beijing, China. The effects of the toll schemes generated from the two models were compared in terms of emissions reduction and congestion mitigation. In this study, it was indicated that a higher total collected toll may lead to more emissions and related treatment costs. A tradeoff existed between the toll scheme, emissions reduction, and congestion mitigation.

Author Contributions: Data curation, software, validation, visualization, and writing-original draft by X.L.; conceptualization, methodology, supervision, and writing—original draft by Y.L.; formal analysis, supervision, and writing - review and editing by W.S.; writing — review and editing by L.Z.

Funding: This research was funded by the National Natural Science Foundation of China (71303017), the Innovative Research Groups of the National Natural Science Foundation of China (71621001), and the Beijing Intelligent Logistics System Collaborative Innovation Center (BILSCIC-2018KF-03).

Acknowledgments: This research was supported by the National Natural Science Foundation of China (71303017), the Innovative Research Groups of the National Natural Science Foundation of China (71621001), and the Beijing Intelligent Logistics System Collaborative Innovation Center (BILSCIC-2018KF-03). The authors thank the anonymous reviewers for their comments and suggestions that helped in improving the manuscript.

Conflicts of Interest: The authors declare no conflicts of interest.

\section{References}

1. European Environment Agency. Emissions of air Pollutants from Transport. Available online: https:/ / www.eea.europa.eu/data-and-maps/indicators/transport-emissions-of-air-pollutants-8/trans port-emissions-of-air-pollutants-6 (accessed on 6 December 2018).

2. Verhoef, E.T. The implementation of marginal external cost pricing in road transport. Pap. Reg. Sci. 2000, 79, 307-332. [CrossRef]

3. Bao, Y.; Gao, Z.; Xu, M.; Yang, H. Tradable credit scheme for mobility management considering travelers' loss aversion. Transp. Res. Part E Logist. Transp. Rev. 2014, 68, 138-154. [CrossRef]

4. He, F.; Yin, Y.; Zhou, J. Deploying public charging stations for electric vehicles on urban road networks. Transp. Res. Part C Emerg. Technol. 2015, 60, 227-240. [CrossRef]

5. Jiang, Y.B.; Sun, H.J.; Wang, W. Congestion pricing and refund optimization model based on mode choice. J. Transp. Syst. Eng. Inf. Technol. 2016, 16, 142-147.

6. Gu, Z.; Liu, Z.; Cheng, Q.; Saberi, M. Congestion pricing practices and public acceptance: A review of evidence. Case Stud. Transp. Policy 2018, 6, 94-101. [CrossRef]

7. Huang, W.J.; Zhang, W.H.; Shen, J.Y.; Jiang, N. Road Congestion Pricing Model Considering Energy Consumption Measurement. J. Transp. Syst. Eng. Inf. Technol. 2018, 18, 166-172.

8. Wang, Y.; Szeto, W.Y.; Han, K.; Friesz, T.L. Dynamic traffic assignment: A review of the methodological advances for environmentally sustainable road transportation applications. Transp. Res. Part B Methodol. 2018, 111, 370-394. [CrossRef]

9. Palma, A.D.; Lindsey, R. Traffic congestion pricing methodologies and technologies. Transp. Res. Part C Emerg. Technol. 2011, 19, 1377-1399. [CrossRef]

10. Wang, G.M.; Gao, Z.Y.; Xu, M. Integrating link-based discrete credit charging scheme into discrete network design problem. Eur. J. Oper. Res. 2019, 272, 176-187. [CrossRef] 
11. Liu, Z.; Meng, Q.; Wang, S. Variational inequality model for cordon-based congestion charging under side constrained stochastic user equilibrium conditions. Transp. A Transp. Sci. 2014, 10, 693-704.

12. Gu, Z.Y.; Shafiei, S.; Liu, Z.Y.; Saberi, M. Optimal distance- and time-dependent area-based pricing with the Network Fundamental Diagram. Transp. Res. Part C Emerg. Technol. 2018, 95, 1-28. [CrossRef]

13. Daganzo, C.F.; Lehe, L.J. Distance-dependent congestion pricing for downtown zones. Transp. Res. Part B Methodol. 2015, 75, 89-99. [CrossRef]

14. Zheng, N.; Rérat, G.; Geroliminis, N. Time-dependent area-based pricing for multimodal systems with heterogeneous users in an agent-based environment. Transp. Res. Part C Emerg. Technol. 2016, 62, 133-148. [CrossRef]

15. Yang, H.; Lam, W. Optimal road tolls under conditions of queueing and congestion. Transp. Res. Part A Policy Pract. 1996, 30, 319-332.

16. Kolak, O.İ; Feyzioğlu, O.; Noyan, N. Bi-level multi-objective traffic network optimization with sustainability perspective. Expert Syst. Appl. 2018, 104, 294-306. [CrossRef]

17. Liu, Z.; Wang, S.; Meng, Q. Optimal joint distance and time toll for cordon-based congestion pricing. Transp. Res. Part B Methodol. 2014, 69, 81-97. [CrossRef]

18. Grisolía, J.M.; López, F.; Ortúzar, J.D. Increasing the acceptability of a congestion charging scheme. Transp. Policy 2015, 39, 37-47.

19. Anas, A.; Hiramatsu, T. The economics of cordon tolling: General equilibrium and welfare analysis. Econ. Transp. 2013, 2, 18-37. [CrossRef]

20. Aziz, H.M.A.; Ukkusuri, S.V. Exploring the trade-off between greenhouse gas emissions and travel time in daily travel decisions: Route and departure time choices. Transp. Res. Part D Transp. Environ. 2014, 32, 334-353. [CrossRef]

21. Li, Z.C.; Guo, Q.W. Optimal time for implementing cordon toll pricing scheme in a monocentric city. Pap. Reg. Sci. 2017, 96, 163-190. [CrossRef]

22. Azari, K.A.; Arintono, S.; Hamid, H.; Davoodi, S.R. Evaluation of demand for different trip purposes under various congestion pricing scenarios. J. Transp. Geogr. 2013, 29, 43-51. [CrossRef]

23. Li, Z.; Lam, W.; Wang, S.; Sumalee, A. Environmentally Sustainable Toll Design for Congested Road Networks with Uncertain Demand. Int. J. Sustain. Transp. 2012, 6, 127-155. [CrossRef]

24. Szeto, W.Y.; Wang, Y.; Wong, S.C. The chemical reaction optimization approach to solving the environmentally sustainable network design problem. Comput. Aided Civ. Infrastruct. Eng. 2014, 29, 140-158. [CrossRef]

25. Ding, D.; Shuai, B.; Zhi, J.C. Impact of Emission Charging to Carpooling in Urban Roads. J. Transp. Syst. Eng. Inf. Technol. 2015, 15, 26-32.

26. Ma, R.; Ban, X.; Szeto, W. Emission modeling and pricing on single-destination dynamic traffic networks. Transp. Res. Part B Methodol. 2017, 100, 255-283. [CrossRef]

27. Long, J.; Chen, J.; Szeto, W.; Shi, Q. Link-based system optimum dynamic traffic assignment problems with environmental objectives. Transp. Res. Part D Transp. Environ. 2018, 60, 56-75. [CrossRef]

28. Schrank, D.; Lomax, T. The 2005 Urban Mobility Report; Texas Transportation Institute: College Station, TX, USA, 2005.

29. Das, D.K.; Keetse, M.S.M. Assessment of traffic congestion in the central areas of Kimberley city. Interim Interdiscip. J. 2015, 14, 70-82.

30. Bian, C.; Yuan, C.; Kuang, W.; Wu, D. Evaluation, Classification, and Influential Factors Analysis of Traffic Congestion in Chinese Cities Using the Online Map Data. Math. Probl. Eng. 2016, 2016, 1693729. [CrossRef]

31. He, F.F.; Yan, X.D.; Liu, Y.; Ma, L. A Traffic Congestion Assessment Method for Urban Road Networks Based on Speed Performance Index. Procedia Eng. 2016, 137, 425-433. [CrossRef]

32. BTMB. Urban Road Traffic Performance Index; Beijing Municipal Commission of Transport: Beijing, China, 2011. (In Chinese)

33. Lv, Y.; Wang, S.S.; Gao, Z.Y.; Li, X.J.; Sun, W. Design of a heuristic environment-friendly road pricing scheme for traffic emission control under uncertainty. J. Environ. Manag. 2018. [CrossRef]

34. Sun, H.; Gao, Z.; Wu, J. A bi-level programming model and solution algorithm for the location of logistics distribution centers. Appl. Math. Model. 2008, 32, 610-616. [CrossRef] 
35. Yin, Y.; Lawphongpanich, S. Internalizing emission externality on road networks. Transp. Res. Part D Transp. Environ. 2006, 11, 292-301. [CrossRef]

36. Beijing Transport Institute. Beijing Transport Development Annual Report. Available online: http://www.bj trc.org.cn/JGJS.aspx?id=5.2\&Menu=GZCG (accessed on 6 December 2018). (In Chinese) 